Article

\title{
Unification of Thermo Field Kinetic and Hydrodynamics Approaches in the Theory of Dense Quantum-Field Systems
}

\author{
Mykhailo Tokarchuk $^{1,2, *}$ and Petro Hlushak ${ }^{1}$ \\ 1 Institute for Condensed Matter Physics of the National Academy of Sciences of Ukraine, 1, Svientsitskii Str., \\ 79011 Lviv, Ukraine; phl@icmp.lviv.ua \\ 2 National University “Lvivska Politechnika”, 12, S. Bandera Str., 79013 Lviv, Ukraine \\ * Correspondence: mtok@icmp.lviv.ua; Tel.: +38-032-276-1978
}

Received: 29 October 2018; Accepted: 13 December 2018; Published: 21 December 2018

\begin{abstract}
A formulation of nonequilibrium thermo-field dynamics has been performed using the nonequilibrium statistical operator method by D.N. Zubarev. Generalized transfer equations for a consistent description of the kinetics and hydrodynamics of the dense quantum field system with strongly-bound states are derived.
\end{abstract}

Keywords: nonequilibrium thermo-field dynamics; kinetics; hydrodynamics; kinetic equations; transport coefficients; bound states; quark-gluon plasma

\section{Introduction}

The problem of accounting for the bound states (clusters) [1,2] formed by particles is particularly important in the development of the theories of nonequilibrium processes of thermal quantum field systems, such as nuclear matter [3-16]. Kinetic and hydrodynamic processes in a hot, compressed nuclear matter, which appears after ultrarelativistic collisions of heavy nuclei $[7,14,16-21]$, are mutually connected, and therefore, the bound states between nucleons should be considered. This is of great importance for the analysis and correlation of final reaction products. Obviously, a nucleon-nucleon interaction investigation based on a quark-gluon plasma is a sequential microscopic approach to the dynamical description of reactions in a nuclear matter. The problems of a dense quark-gluon matter were discussed in detail in $[4,5,12,13,22-27]$.

In his recent works $[1,2,19]$, G. Röpke noted the importance of constructing a nonequilibrium theory in which along with hydrodynamic parameters, a cluster distribution function is taken into account, similarly to the case of the classical theory of non-equilibrium processes of dense gases and liquids [28-31].

In modern theoretical studies of the nonequilibrium properties of quark-gluon plasma $[12,13,23,24]$, which is one of the states of nuclear matter, one of the most widely-used statistical concepts is the entropy of Tsallis and Renyi [32-42]. This is due to the fact that the results of experimental data on the distribution of high-energy hadrons over transverse momentums [32] are described by the power distributions and are characterized by temperature oscillations and possible fractal structures [43]. At the same time, the important problem of the construction of kinetic and hydrodynamic equations for nuclear matter of high density and high temperature is not sufficiently addressed for these systems. However, within the framework of the Gibbs statistics, the equations of hydrodynamics and thermodynamics were already considered in many papers using the method of Zubarev's nonequilibrium statistical operator [44-53], the projection operator method [54,55], and kinetic equations [56-59]. Thus, we propose an approach to solve these problems based on the nonequilibrium 
thermo-field dynamics [60-62] in the formulation of the method of the nonequilibrium statistical operator [63-65]. We use the thermo-field formalism proposed by Umezawa, Matsumoto, and Tachiki [66,67], mainly because it accounts for the quantum field nature of the interaction of the particles in the synthesis with Gibbs statistics.

Below, in the Section 2 of this paper, we consider the nonequilibrium thermo-field dynamics in the formulation of the nonequilibrium statistical operator method [68-71] in Renyi statistics. We use the statistics of Renyi, which has the power distribution. When the Renyi parameter is $q=1$, we obtain the results of Gibbs statistics. In addition, Renyi entropy is additive in contrast to the Tsallis entropy.

Next, in the Section 3, generalized equations for the consistent description of kinetic and hydrodynamic processes, which take into account the bound states that emerge in the thermal quantum field system, will be presented.

\section{Nonequilibrium Statistical Operator in Thermo-Field Space}

We use the nonequilibrium statistical operator method in the thermo-field formulation [63,64], where the mean values corresponding to the observables can be found using the nonequilibrium thermo-vacuum state vector $|\varrho(t)\rangle\rangle$ :

$$
\langle A\rangle^{t}=\langle\langle 1 \mid A \varrho(t)\rangle\rangle=\langle\langle 1|\hat{A}| \varrho(t)\rangle\rangle,
$$

where $\hat{A}$ is a superoperator acting on the state $|\varrho(t)\rangle\rangle$. The nonequilibrium thermo-vacuum state vector $|\varrho(t)\rangle\rangle$ satisfies the Schrödinger equation [63]:

$$
\left.\left.\frac{\partial}{\partial t}|\varrho(t)\rangle\right\rangle-\left|\frac{1}{i \hbar}[H, \varrho(t)]\right\rangle\right\rangle=0,
$$

or:

$$
\left.\left.\frac{\partial}{\partial t}|\varrho(t)\rangle\right\rangle-\frac{1}{i \hbar} \hat{H}|\varrho(t)\rangle\right\rangle=0 .
$$

Here, the total Hamiltonian $\hat{H}$ takes the form:

$$
\hat{H}=H-\tilde{H},
$$

where $\left\langle\langle 1| \hat{H}=0\right.$ and $H=H\left(\hat{a}^{+}, \hat{a}\right), \tilde{H}=H^{(*)}\left(\tilde{a}^{+}, \tilde{a}\right)$ are superoperators constructed from the creation and annihilation of superoperators of the thermal Liouville space $[63,66,67]$. The superoperators $H$ and $\tilde{H}$ are accordingly defined by the relations:

$$
|H \varrho(t)\rangle\rangle=H|\varrho(t)\rangle\rangle, \quad|\varrho(t) H\rangle\rangle=\tilde{H}|\varrho(t)\rangle\rangle .
$$

The superoperators $\hat{a}_{l}^{+}, \hat{a}_{j}, \tilde{a}_{l}^{+}$and $\tilde{a}_{j}$ satisfy the same commutation relations as the operators $a_{l}^{+}, a_{j}$ of the corresponding statistics:

$$
\begin{array}{ll}
{\left[\hat{a}_{l}, \hat{a}_{j}^{+}\right]_{\sigma}=\left[\tilde{a}_{l}, \tilde{a}_{j}^{+}\right]_{\sigma}=\delta_{l j,}} & {\left[\hat{a}_{l}, \tilde{a}_{j}\right]_{\sigma}=\left[\hat{a}_{l}^{+}, \tilde{a}_{j}^{+}\right]_{\sigma}=0,} \\
{\left[\hat{a}_{l}, \hat{a}_{j}\right]_{\sigma}=\left[\hat{a}_{l}^{+}, \hat{a}_{j}^{+}\right]_{\sigma}=0,} & {\left[\tilde{a}_{l}, \tilde{a}_{j}\right]_{\sigma}=\left[\tilde{a}_{l}^{+}, \tilde{a}_{j}^{+}\right]_{\sigma}=0,}
\end{array}
$$

where $[A, B]_{\sigma}=A B-\sigma B A, \sigma=+1$ for bosons and $\sigma=-1$ for fermions. The annihilation superoperators $\hat{a}_{l}$ and $\tilde{a}_{l}$ are defined by their action on the ground state, the supervacuum [60-62]:

$$
\left.\left.\hat{a}_{l}|00\rangle\right\rangle=\tilde{a}_{l}|00\rangle\right\rangle=0,
$$

where $|00\rangle\rangle=|0\rangle\langle 0 \mid\rangle\rangle$ is the supervacuum. In this case, we have the relations $\hat{a}_{l}|0\rangle=a_{l}|0\rangle=0$ and $\langle 0| \tilde{a}_{l}=0$. In other words, the supervacuum $\left.|00\rangle\right\rangle$ is the orthogonalized state of two vacuum states $\langle 0|$ 
and $|0\rangle$. Taking commutation relations (6) and definitions (7) into account, we can represent the unit vectors $\left.\left.|1\rangle\rangle=\left|\sum_{l}\right| l\right\rangle\langle l \mid\rangle\right\rangle$ and $\left\langle\langle 1|=\left\langle\left\langle\sum_{l} \mid l\right\rangle\langle l||\right.\right.$ in the form

$$
\begin{aligned}
& \left.|1\rangle\rangle=\exp \left\{\sum_{l} \hat{a}_{l}^{+} \tilde{a}_{l}^{+}\right\}|00\rangle\right\rangle, \\
& \left\langle\langle 1|=\left\langle\langle 00| \exp \left\{\sum_{l} \tilde{a}_{l} \hat{a}_{l}\right\} .\right.\right.
\end{aligned}
$$

Using these vectors, we write the relations between the actions of the superoperators $\hat{a}_{l}^{+}, \hat{a}_{j}, \tilde{a}_{l}^{+}$, and $\tilde{a}_{j}$ :

$$
\begin{aligned}
\left.\left.\hat{a}_{l}|1\rangle\right\rangle=\tilde{a}_{l}^{+}|1\rangle\right\rangle, \quad & \left\langle\langle 1| \hat{a}_{l}^{+}=\left\langle\langle 1| \tilde{a}_{l},\right.\right. \\
\left.\left.\hat{a}_{l}^{+}|1\rangle\right\rangle=\sigma \tilde{a}_{l}|1\rangle\right\rangle, & \left\langle\langle 1| \hat{a}_{l}=\left\langle\langle 1| \tilde{a}_{l}^{+} \sigma .\right.\right.
\end{aligned}
$$

Hence, in the thermo-field dynamics formalism [60-62], the number of operators is doubled by introducing the operators $A\left(\hat{a}^{+}, \hat{a}\right)$ and $\tilde{A}\left(\tilde{a}^{+}, \tilde{a}\right)$, which satisfy the conditions:

$$
\begin{aligned}
\widetilde{A_{1} A_{2}} & =\tilde{A}_{1} \tilde{A}_{2}, \quad \tilde{\tilde{A}}=A, \\
c_{1} A_{1}+c_{2} A_{2} & =c_{1}^{*} \tilde{A}_{1}+c_{2}^{*} \tilde{A}_{2}, \\
|A\rangle\rangle & =\hat{A}|1\rangle\rangle, \\
\left.\left|A_{1} A_{2}\right\rangle\right\rangle & \left.=\hat{A}_{1}\left|A_{2}\right\rangle\right\rangle,
\end{aligned}
$$

where the asterisk denotes complex conjugation. A more detailed description of the properties of the superoperators $\hat{a}_{l}^{+}, \hat{a}_{j}, \tilde{a}_{l}^{+}$, and $\tilde{a}_{j}$ and the thermal Liouville space was presented in [60-62]. The nonequilibrium thermo vacuum state vector is normalized: $\langle\langle 1 \mid \varrho(t)\rangle\rangle=\langle\langle 1|\hat{\varrho}(t)| 1\rangle\rangle=1$, where $\hat{\varrho}(t)$ is the nonequilibrium statistical superoperator, which depends on $\hat{a}_{l}^{+}$and $\hat{a}_{j}, \hat{\varrho}(t) \equiv \varrho\left(\hat{a}^{+}, \hat{a} ; t\right)$. The superoperator $\tilde{\varrho}(t) \equiv \varrho^{+}\left(\tilde{a}^{+}, \tilde{a} ; t\right)$ depends on $\tilde{a}_{l}^{+}, \tilde{a}_{j}$.

In the nonequilibrium statistical operator method in the thermo-field formulation $[63,64]$, the nonequilibrium thermo-vacuum state vector as a solution of the Schrödinger Equation (3) with a source $\left.\left.-\varepsilon(|\varrho(t)\rangle\rangle-\left|\varrho_{\text {rel }}(t)\right\rangle\right\rangle\right)$, with the projection taken into account, can be found in the form:

$$
\left.\left.|\varrho(t)\rangle\rangle=\left|\varrho_{\text {rel }}(t)\right\rangle\right\rangle+\int_{-\infty}^{t} d t^{\prime} \mathrm{e}^{\varepsilon\left(t^{\prime}-t\right)} T\left(t, t^{\prime}\right)\left[1-\mathcal{P}_{\text {rel }}\left(t^{\prime}\right)\right] \frac{1}{i \hbar} \hat{H}\left|\varrho_{\text {rel }}\left(t^{\prime}\right)\right\rangle\right\rangle .
$$

Here, $T\left(t, t^{\prime}\right)=\exp _{+}\left\{\int_{t^{\prime}}^{t} d t^{\prime}\left[1-\mathcal{P}_{\text {rel }}\left(t^{\prime}\right)\right] \frac{1}{\mathrm{i} \hbar} \hat{H}\right\}$ is the evolution operator with the projection taken into account, where $\exp _{+}$is the ordered exponential, $\varepsilon \rightarrow+0$ after the thermodynamic limit transition.

$$
\begin{aligned}
\left.\left.\mathcal{P}_{\text {rel }}(t)(|\ldots\rangle\rangle\right)=\left|\varrho_{\text {rel }}(t)\right\rangle\right\rangle & +\sum_{n} \frac{\left.\delta\left|\varrho_{\text {rel }}(t)\right\rangle\right\rangle}{\delta\left\langle\left\langle 1\left|\hat{p}_{n}\right| \varrho(t)\right\rangle\right\rangle}\left\langle\left\langle 1\left|\hat{p}_{n}\right| \ldots\right\rangle\right\rangle \\
& -\sum_{n} \frac{\left.\delta\left|\varrho_{\text {rel }}(t)\right\rangle\right\rangle}{\delta\left\langle\left\langle 1\left|\hat{p}_{n}\right| \varrho(t)\right\rangle\right\rangle}\left\langle\left\langle 1\left|\hat{p}_{n}\right| \ldots\right\rangle\right\rangle\langle\langle 1 \mid \ldots\rangle\rangle
\end{aligned}
$$

is the Kawasaki-Ganton projection operator, which acts only on the state vectors $|\ldots\rangle\rangle$ and has the operator properties $\left.\left.\left.\left.\mathcal{P}_{\text {rel }}(t)\left|\varrho\left(t^{\prime}\right)\right\rangle\right\rangle=\left|\varrho_{\text {rel }}(t)\right\rangle\right\rangle, \mathcal{P}_{\text {rel }}(t)\left|\varrho_{\text {rel }}\left(t^{\prime}\right)\right\rangle\right\rangle=\left|\varrho_{\text {rel }}(t)\right\rangle\right\rangle, \mathcal{P}_{\text {rel }}(t) \mathcal{P}_{\text {rel }}\left(t^{\prime}\right)=\mathcal{P}_{\text {rel }}(t)$. The relevant thermo-vacuum state vector $\left.\left.\left|\varrho_{\text {rel }}(t)\right\rangle\right\rangle=\hat{\varrho}_{\text {rel }}(t)|1\rangle\right\rangle$, is normalized in accordance with the relation $\left\langle\left\langle 1 \mid \varrho_{\text {rel }}(t)\right\rangle\right\rangle=\left\langle\left\langle 1\left|\hat{\varrho}_{\text {rel }}(t)\right| 1\right\rangle\right\rangle=1$, where $\hat{\varrho}_{\text {rel }}(t)$ is the relevant statistical superoperator. The relevant thermo-vacuum state vector of the system can be defined as follows. We assume that $\left\langle p_{n}\right\rangle^{t}=\left\langle\left\langle 1\left|\hat{p}_{n}\right| \varrho(t)\right\rangle\right\rangle$ is the set of observed variables describing the nonequilibrium system state, where $p_{n}$ are the operators constructed on the respective creation and annihilation operators $a_{l}^{+}$and $a_{l}$. The relevant statistical operator $\varrho_{\text {rel }}(t)$ is determined from the extremum of the Renyi entropy functional:

$$
L_{R}(t)=\frac{1}{1-q} \ln \left\langle\left\langle 1 \mid\left(\left|\varrho^{\prime}(t)\right\rangle\right\rangle\right)^{q}-\alpha\left\langle\left\langle 1 \mid \varrho^{\prime}(t)\right\rangle\right\rangle-\sum_{n} F_{n}^{*}(t)\left\langle\left\langle 1\left|\hat{p}_{n}\right| \varrho^{\prime}(t)\right\rangle\right\rangle\right.
$$


under the additional condition that the mean values $\left\langle p_{n}\right\rangle^{t}$ are given with the normalization condition $\langle\langle 1|\hat{\varrho}(t)| 1\rangle\rangle=1$ preserved. The Lagrange parameters $\alpha$ and $F_{n}^{*}(t)$ are determined from the respective normalization condition and self-consistency conditions:

$$
\langle\ldots\rangle_{\text {rel }}^{t}=\left\langle\left\langle 1|\ldots| \varrho_{\text {rel }}(t)\right\rangle\right\rangle, \quad\left\langle p_{n}\right\rangle^{t}=\left\langle p_{n}\right\rangle_{\text {rel }}^{t}=\left\langle\left\langle 1\left|\hat{p}_{n}\right| \varrho_{\text {rel }}(t)\right\rangle\right\rangle .
$$

The relevant statistical operator $\varrho_{\text {rel }}(t)$ then becomes:

$$
\varrho_{\text {rel }}(t)=\frac{1}{Z_{R}(t)}\left[1-\frac{q-1}{q} \sum_{n} F_{n}^{*}(t) \delta \hat{p}_{n}(t)\right]^{\frac{1}{q-1}},
$$

where $q$ is the Renyi parameter, $\delta \hat{p}_{n}(t)=\hat{p}-\left\langle\left\langle 1\left|\hat{p}_{n}\right| \varrho(t)\right\rangle\right\rangle$, and:

$$
Z_{R}(t)=\left\langle\left\langle 1 \mid\left[1-\frac{q-1}{q} \sum_{n} F_{n}^{*}(t) \delta \hat{p}_{n}(t)\right]^{\frac{1}{q-1}}\right\rangle\right\rangle,
$$

is the partition function. The sum over $n$ can denote the summation over the wave vector $\mathbf{k}$, the kind of particles, and a whole series of quantum numbers, such as spin. From (14) at $q=1$, we obtain the relevant statistical operator corresponding to Gibbs statistics [63]:

$$
\varrho_{\text {rel }}(t)=\exp \left\{-\Phi(t)-\sum_{n} F_{n}^{*}(t) p_{n}\right\},
$$

where $\Phi(t)=\ln S p \exp \left\{-\sum_{n} F_{n}^{*}(t) p_{n}\right\}$ is the Massieu-Planck functional. Substituting (14) in (11), we now obtain the nonequilibrium thermo-vacuum vector:

$$
\left.\left.|\varrho(t)\rangle\rangle=\left|\varrho_{\text {rel }}(t)\right\rangle\right\rangle+\sum_{n} \int_{-\infty}^{t} d t^{\prime} e^{\varepsilon\left(t^{\prime}-t\right)} T\left(t, t^{\prime}\right)\left|\int_{0}^{1} d \tau \varrho_{\text {rel }}^{\tau}\left(t^{\prime}\right) J_{n}\left(t^{\prime}\right) \varrho_{\text {rel }}(t)^{1-\tau}\left(t^{\prime}\right)\right\rangle\right\rangle F_{n}^{*}\left(t^{\prime}\right),
$$

where $J_{n}(t)=[1-\mathcal{P}(t)] \frac{1}{q} \psi^{-1}(t) \dot{\hat{p}}_{n}$ are the operators of the generalized flows describing the dissipative processes $\dot{\hat{p}}_{n}=-\frac{1}{\mathrm{i} \hbar} \hat{H} \hat{p}_{n}$ in the system. The projection operator $\mathcal{P}(t)$ acts on operators and has the structure:

$$
\begin{gathered}
\mathcal{P}(t)(\ldots)=\left\langle\left\langle 1|\ldots| \varrho_{\text {rel }}(t)\right\rangle\right\rangle+\sum_{m} \delta\left[\int _ { 0 } ^ { 1 } d \tau \varrho _ { \text { rel } } ^ { \tau } ( t ) \psi ^ { - 1 } ( t ) \left(F_{m}(t)\right.\right. \\
\left.\left.+\sum_{n} f_{m n}^{-1}(t) \delta \hat{p}_{n}\right) \varrho_{\text {rel }}^{-\tau}\right]\left\langle\left\langle\ldots \mid \int_{0}^{1} d \tau \varrho_{\text {rel }}^{\tau}(t) \delta \hat{p}_{n} \varrho_{\text {rel }}^{-\tau}(t) \varrho_{\text {rel }}(t)\right\rangle\right\rangle,
\end{gathered}
$$

where $\delta[\ldots]=[\ldots]-\left\langle\left\langle 1|[\ldots]| \varrho_{\text {rel }}(t)\right\rangle\right\rangle$ and $f_{m n}(t)=\frac{\delta\left\langle\left\langle 1\left|\hat{p}_{m}\right| \varrho(t)\right\rangle\right.}{\delta F_{n}(t)}$. The operator $\psi(t)$ has the form $\psi(t)=$ $1-\frac{q-1}{q} \sum_{n} F_{n}^{*}(t) \delta \hat{p}_{n}(t)$. Using the nonequilibrium thermo-vacuum state vector $\left.|\varrho(t)\rangle\right\rangle$ given by (17), we obtain the transport equations for the nonequilibrium means $\left\langle\left\langle 1\left|\hat{p}_{n}\right| \varrho(t)\right\rangle\right\rangle$ in the thermo-field representation. For this, we use the identity:

$$
\frac{\partial}{\partial t}\left\langle\left\langle 1\left|\hat{p}_{n}\right| \varrho(t)\right\rangle\right\rangle=\left\langle\left\langle 1\left|\dot{\hat{p}}_{n}\right| \varrho(t)\right\rangle\right\rangle=\left\langle\left\langle 1\left|\dot{\hat{p}}_{n}\right| \varrho_{\text {rel }}(t)\right\rangle\right\rangle+\left\langle\left\langle J_{n}(t) \mid \varrho(t)\right\rangle\right\rangle .
$$

Averaging the last term on the right-hand side with $|\varrho(t)\rangle\rangle$ given by (17), we obtain the transport equations for the means $\left\langle\left\langle 1\left|\hat{p}_{n}\right| \varrho_{\text {rel }}(t)\right\rangle\right\rangle$ :

$$
\begin{aligned}
\frac{\partial}{\partial t}\left\langle\left\langle 1\left|\hat{p}_{n}\right| \varrho(t)\right\rangle\right\rangle= & \left\langle\left\langle 1\left|\dot{\hat{p}}_{n}\right| \varrho_{\text {rel }}(t)\right\rangle\right\rangle \\
& +\sum_{n^{\prime}} \int_{-\infty}^{t} d t^{\prime} e^{\varepsilon\left(t^{\prime}-t\right)}\left\langle\left\langle\dot{\hat{p}}_{n} T\left(t, t^{\prime}\right) \mid \int_{0}^{1} d \tau \varrho_{\text {rel }}^{\tau}\left(t^{\prime}\right) J_{n^{\prime}}\left(t^{\prime}\right) \varrho_{\text {rel }}^{1-\tau}\left(t^{\prime}\right)\right\rangle\right\rangle F_{n^{\prime}}^{*}\left(t^{\prime}\right) .
\end{aligned}
$$


Transport Equation (20) takes the memory effects into account and can be used to describe nonequilibrium processes in quantum Bose and Fermi systems in concrete cases in the framework of the nonequilibrium thermo-field dynamics of extensive statistics. In particular, a system of relativistic transport equations for a consistent description of the kinetic and hydrodynamic processes in a quark-gluon system was derived in [64] using the nonequilibrium statistical operator method in the thermo-field representation in Gibbs statistics. The advanced approach in terms of Renyi statistics can be generalized to the case of relativistic systems, and this observation is important [33,35-42]. This subject will be described in forthcoming works.

\section{Thermo-Field Transport Equation Taking into Account Bound States}

We will consider a quantum field system in which bound states can appear between the particles. Let us introduce annihilation and creation operators of a bound state $(A \alpha)$ with the $A$-particle:

$$
\begin{aligned}
& a_{A \alpha}(\mathbf{p})=\sum_{1, \ldots, A} \Psi_{A \alpha \mathbf{p}}(1, \ldots, A) a(1) \ldots a(A) \\
& a_{A \alpha}^{+}(\mathbf{p})=\sum_{1, \ldots, A} \Psi_{A \alpha \mathbf{p}}^{*}(1, \ldots, A) a^{+}(1) \ldots a^{+}(A),
\end{aligned}
$$

where $\Psi_{A \alpha \mathbf{p}}(1, \ldots, A)$ is a self-function of the $A$-particle bound state, $\alpha$ denotes internal quantum numbers (spin, etc.), $\mathbf{p}$ is a particle momentum, and the sum covers the particles. Annihilation and creation operators $a(j)$ and $a^{+}(j)$ satisfy the following commutation relations:

$$
\left[a(l), a^{+}(j)\right]_{\sigma}=\delta_{l, j}, \quad[a(l), a(j)]_{\sigma}=\left[a^{+}(l), a^{+}(j)\right]_{\sigma}=0,
$$

where the $\sigma$-commutator is determined by $[a, b]_{\sigma}=a b-\sigma b a$ with $\sigma= \pm 1:+1$ for bosons and -1 for fermions.

The Hamiltonian of such a system can be written in the form:

$$
\begin{gathered}
H=\sum_{A, \alpha} \int \frac{\mathrm{d} \mathbf{p d} \mathbf{q}}{(2 \pi \hbar)^{6}} \frac{p^{2}}{2 m_{A}} a_{A \alpha}^{+}\left(\mathbf{p}-\frac{\mathbf{q}}{2}\right) a_{A \alpha}\left(\mathbf{p}+\frac{\mathbf{q}}{2}\right) \\
+\frac{1}{2} \sum_{A, B} \sum_{\alpha, \beta} \int \frac{\mathrm{d} \mathbf{p d} \mathbf{p}^{\prime} \mathrm{d} \mathbf{q}}{(2 \pi \hbar)^{9}} V_{A B}(\mathbf{q}) a_{A \alpha}^{+}\left(\mathbf{p}+\frac{\mathbf{q}-\mathbf{p}^{\prime}}{2}\right) \hat{n}_{B \beta}(\mathbf{q}) a_{A \alpha}\left(\mathbf{p}-\frac{\mathbf{q}-\mathbf{p}^{\prime}}{2}\right),
\end{gathered}
$$

where $V_{A B}(\mathbf{q})$ is interaction energy between $A$ - and $B$-particle bound states and $\mathbf{q}$ is a wavevector. Annihilation and creation operators $a_{A \alpha}(\mathbf{p})$ and $a_{A \alpha}^{+}(\mathbf{p})$ satisfy the following commutation relations:

$$
\begin{aligned}
& {\left[a_{A \alpha}(\mathbf{p}), a_{B \beta}^{+}\left(\mathbf{p}^{\prime}\right)\right]_{\sigma}=\delta_{A, B} \delta_{\alpha, \beta} \delta\left(\mathbf{p}-\mathbf{p}^{\prime}\right),} \\
& {\left[a_{A \alpha}(\mathbf{p}), a_{B \beta}\left(\mathbf{p}^{\prime}\right)\right]_{\sigma}=\left[a_{A \alpha}^{+}(\mathbf{p}), a_{B \beta}^{+}\left(\mathbf{p}^{\prime}\right)\right]_{\sigma}=0 .}
\end{aligned}
$$

$\hat{n}_{B \beta}(\mathbf{q})$ in (23) is a Fourier transform of the $B$-particle density operator:

$$
\hat{n}_{B \beta}(\mathbf{q})=\int \frac{\mathrm{d} \mathbf{p}}{(2 \pi \hbar)^{3}} a_{B \mathbf{p}-\frac{\mathbf{q}}{2}}^{+} a_{B \mathbf{p}+\frac{\mathbf{q}}{2}} .
$$

As parameters of a reduced description for the consistent description of the kinetics and hydrodynamics of a system, where bound states between the particles can appear, let us choose nonequilibrium distribution functions of $A$-particle bound states in thermo-field representation:

$$
\left\langle\left\langle 1\left|\hat{n}_{A \alpha}(\mathbf{r}, \mathbf{p})\right| \varrho(t)\right\rangle\right\rangle=f_{A \alpha}(\mathbf{r}, \mathbf{p} ; t)=f_{A \alpha}(x ; t), \quad x=\{\mathbf{r}, \mathbf{p}\} .
$$


Here, $f_{A \alpha}(x ; t)$ is a Wigner function of the $A$-particle bound state where:

$$
\hat{n}_{A \alpha}(\mathbf{r}, \mathbf{p}) \equiv \hat{n}_{A \alpha}(x)=\int \frac{\mathrm{d} \mathbf{q}}{(2 \pi \hbar)^{3}} \mathrm{e}^{-\frac{1}{i} \mathbf{q} \cdot \mathbf{r}} \hat{a}_{A \alpha}^{+}\left(\mathbf{p}-\frac{\mathbf{q}}{2}\right) \hat{a}_{A \alpha}\left(\mathbf{p}+\frac{\mathbf{q}}{2}\right)
$$

is the Klimontovich density operator; and the average value of the total energy density operator:

$$
\langle\langle 1|\hat{H}(\mathbf{r})| \varrho(t)\rangle\rangle=\langle\langle 1 \mid H(\mathbf{r}) \varrho(t)\rangle\rangle .
$$

By this $\int \mathrm{d} \mathbf{r} H(\mathbf{r})=H, \hat{H}(\mathbf{r})$ is a superoperator of the total energy density, which is constructed on annihilation and creation superoperators $\hat{a}_{A \alpha}(\mathbf{p})$ and $\hat{a}_{A \alpha}^{+}(\mathbf{p})$. The latter satisfy commutation relations (24). Following [63], one can rewrite relevant statistical operator $\left.\left.\hat{\varrho}_{\text {rel }}(t),\left|\varrho_{\text {rel }}(t)\right\rangle\right\rangle=\hat{\varrho}_{\text {rel }}(t)|1\rangle\right\rangle$ and with (14) from $q=1$ for the mentioned parameters of a reduced description in the form:

$$
\hat{\varrho}_{r e l}(t)=\exp \left\{-\Phi^{*}(t)-\int \mathrm{d} \mathbf{r} \beta(\mathbf{r} ; t)\left(\hat{H}(\mathbf{r})-\sum_{A, \alpha} \int \frac{\mathrm{d} \mathbf{p}}{(2 \pi \hbar)^{3}} \mu_{A \alpha}(x ; t) \hat{n}_{A \alpha}(x)\right)\right\},
$$

where Lagrange multipliers $\beta(\mathbf{r} ; t)$ and $\mu_{A \alpha}(x ; t)$ can be found from the self-consistency conditions, respectively:

$$
\begin{aligned}
\langle\langle 1|\hat{H}(\mathbf{r})| \varrho(t)\rangle\rangle & =\left\langle\left\langle 1|\hat{H}(\mathbf{r})| \varrho_{\text {rel }}(t)\right\rangle\right\rangle, \\
\left\langle\left\langle 1\left|\hat{n}_{A \alpha}(x)\right| \varrho(t)\right\rangle\right\rangle & =\left\langle\left\langle 1\left|\hat{n}_{A \alpha}(x)\right| \varrho_{r e l}(t)\right\rangle\right\rangle,
\end{aligned}
$$

$\Phi^{*}(t)$ is the Massieu-Planck functional, and it can be defined from the normalization condition:

$$
\Phi^{*}(t)=\ln \left\langle\left\langle 1 \mid \exp \left\{-\int \mathrm{d} \mathbf{r} \beta(\mathbf{r} ; t)\left(\hat{H}(\mathbf{r})-\sum_{A, \alpha} \int \frac{\mathrm{d} \mathbf{p}}{(2 \pi \hbar)^{3}} \mu_{A \alpha}(x ; t) \hat{n}_{A \alpha}(x)\right)\right\}\right\rangle .\right.
$$

Using now the general structure of nonequilibrium thermo-field dynamics (20), one can obtain a set of generalized transport equations for $A$-particle Wigner distribution functions and the average interaction energy:

$$
\begin{gathered}
\frac{\partial}{\partial t}\left\langle\left\langle 1\left|\hat{n}_{A \alpha}(x)\right| \varrho(t)\right\rangle\right\rangle=\left\langle\left\langle 1\left|\dot{\hat{n}}_{A \alpha}(x)\right| \varrho_{\mathrm{q}}(t)\right\rangle\right\rangle \\
+\int \mathrm{d} \mathbf{r}^{\prime} \int_{-\infty}^{t} \mathrm{~d} t^{\prime} \mathrm{e}^{\varepsilon\left(t^{\prime}-t\right)} \varphi_{n H}^{A \alpha}\left(x, \mathbf{r}^{\prime} ; t, t^{\prime}\right) \beta\left(\mathbf{r}^{\prime} ; t^{\prime}\right) \\
+\sum_{B, \beta} \int \mathrm{d} x^{\prime} \int_{-\infty}^{t} \mathrm{~d} t^{\prime} \mathrm{e}^{\varepsilon\left(t^{\prime}-t\right)} \varphi_{n n}^{A \alpha B \beta}\left(x, x^{\prime} ; t, t^{\prime}\right) \beta\left(\mathbf{r}^{\prime} ; t^{\prime}\right) \mu_{B \beta}\left(x^{\prime} ; t^{\prime}\right), \\
+\int_{-\infty} \mathrm{d} \mathbf{r}^{\prime} \int_{-\infty}^{t} \mathrm{~d} t^{\prime} \mathrm{e}^{\varepsilon\left(t^{\prime}-t\right)} \varphi_{H H}\left(\mathbf{r}, \mathbf{r}^{\prime} ; t, t^{\prime}\right) \beta\left(\mathbf{r}^{\prime} ; t^{\prime}\right) \\
+\sum_{B, \beta} \int \mathrm{d} x^{\prime} \int_{-\infty}^{t} \mathrm{~d} t^{\prime} \mathrm{e}^{\varepsilon\left(t^{\prime}-t\right)} \varphi_{H n}^{B \beta}\left(\mathbf{r}, x^{\prime} ; t, t^{\prime}\right) \beta\left(\mathbf{r}^{\prime} ; t^{\prime}\right) \mu_{B \beta}\left(x^{\prime} ; t^{\prime}\right),
\end{gathered}
$$


where $x^{\prime}=\left\{\mathbf{r}^{\prime}, \mathbf{p}^{\prime}\right\}, d x^{\prime}=(2 \pi \hbar)^{-3} d \mathbf{r}^{\prime} d \mathbf{p}^{\prime}$. Here,

$$
\begin{aligned}
& \varphi_{n n}^{A \alpha}\left(x, x^{\prime} ; t, t^{\prime}\right)=\left\langle\left\langle 1\left|\hat{J}_{n_{A \alpha}}(x, t) T\left(t, t^{\prime}\right)\right| \int_{0}^{1} d \tau \varrho_{r e l}^{\tau}\left(t^{\prime}\right) J_{n_{B \beta}}\left(x^{\prime} ; t^{\prime}\right) \varrho_{r e l}^{1-\tau}\left(t^{\prime}\right)\right\rangle\right\rangle, \\
& \varphi_{n H}^{A \alpha}\left(x, \mathbf{r}^{\prime} ; t, t^{\prime}\right)=\left\langle\left\langle 1\left|\hat{J}_{n_{A \alpha}}(x, t) T\left(t, t^{\prime}\right)\right| \int_{0}^{1} \mathrm{~d} \tau \varrho_{r e l}^{\tau}\left(t^{\prime}\right) J_{H}\left(\mathbf{r}^{\prime} ; t^{\prime}\right) \varrho_{r e l}^{1-\tau}\left(t^{\prime}\right)\right\rangle\right\rangle, \\
& \varphi_{H n}^{B \beta}\left(\mathbf{r}^{\prime}, x^{\prime} ; t, t^{\prime}\right)=\left\langle\left\langle 1\left|\hat{J}_{H}(\mathbf{r}, t) T\left(t, t^{\prime}\right)\right| \int_{0}^{1} \mathrm{~d} \tau \varrho_{r e l}^{\tau}\left(t^{\prime}\right) J_{n_{B \beta}}\left(x^{\prime} ; t^{\prime}\right) \varrho_{r e l}^{1-\tau}\left(t^{\prime}\right)\right\rangle\right\rangle, \\
& \varphi_{H H}\left(\mathbf{r}, \mathbf{r}^{\prime} ; t, t^{\prime}\right)=\left\langle\left\langle 1\left|\hat{J}_{H}(\mathbf{r}, t) T\left(t, t^{\prime}\right)\right| \int_{0}^{1} \mathrm{~d} \tau \varrho_{r e l}^{\tau}\left(t^{\prime}\right) J_{H}\left(\mathbf{r}^{\prime} ; t^{\prime}\right) \varrho_{r e l}^{1-\tau}\left(t^{\prime}\right)\right\rangle\right\rangle
\end{aligned}
$$

are generalized transport cores, which describe dissipative processes. In these formulae:

$$
\begin{array}{ll}
J_{H}(\mathbf{r} ; t) & =\left(1-\mathrm{P}\left(t^{\prime}\right)\right) \dot{H}(\mathbf{r}) \\
J_{n_{A \alpha}}(\mathbf{r}, \mathbf{p} ; t) & =\left(1-\mathrm{P}\left(t^{\prime}\right)\right) \dot{n}_{A \alpha}(x)
\end{array}
$$

are generalized flows, $\dot{H}(\mathbf{r})=-\frac{1}{\mathrm{i} \hbar}[H, H(\mathbf{r})], \dot{n}_{A \alpha}(\mathbf{r}, \mathbf{p})=-\frac{1}{\mathrm{i} \hbar}\left[H, n_{A \alpha}(x)\right]$, and $\mathrm{P}(t)$ is a generalized Mori projection operator in thermo-field representation. It acts on operators:

$$
\begin{aligned}
\mathrm{P}(t) P= & \left\langle\left\langle|\hat{P}| \varrho_{\text {rel }}(t)\right\rangle\right\rangle+\int \mathrm{d} \mathbf{r} \frac{\delta\left\langle\left\langle 1|\hat{P}| \varrho_{\text {rel }}(t)\right\rangle\right\rangle}{\delta\langle\langle 1|\hat{H}(\mathbf{r})| \varrho(t)\rangle\rangle}(H(\mathbf{r})-\langle\langle 1|\hat{H}(\mathbf{r})| \varrho(t)\rangle\rangle) \\
& +\sum_{A, \alpha} \int \frac{\mathrm{d} \mathbf{r} \mathrm{d} \mathbf{p}}{(2 \pi \hbar)^{3}} \frac{\delta\left\langle\left\langle 1|\hat{P}| \varrho_{\text {rel }}(t)\right\rangle\right\rangle}{\delta\left\langle\left\langle 1\left|\hat{n}_{A \alpha}(x)\right| \varrho(t)\right\rangle\right\rangle}\left(n_{A \alpha}(x)-\left\langle\left\langle 1\left|\hat{n}_{A \alpha}(x)\right| \varrho(t)\right\rangle\right\rangle\right)
\end{aligned}
$$

and has all the properties of a projection operator:

$$
\begin{gathered}
\mathrm{P}(t) H(\mathbf{r})=H(\mathbf{r}), \quad \mathrm{P}(t) \mathrm{P}\left(t^{\prime}\right)=\mathrm{P}(t), \\
\mathrm{P}(t) n_{A \alpha}(\mathbf{r}, \mathbf{p})=n_{A \alpha}(\mathbf{r}, \mathbf{p}), \quad(1-\mathrm{P}(t)) \mathrm{P}(t)=0 .
\end{gathered}
$$

The obtained transport equations have the general meaning and can describe both weak and strong nonequilibrium processes of a quantum system taking into consideration bound states. In the calculation of the transport cores (34)-(37) in each case, the problem arises due to the fact that the relevant thermo-vacuum state is not the ground state for the superoperators $\hat{a}_{A \alpha}(\mathbf{P}), \hat{a}_{A \alpha}^{+}(\mathbf{P})$ and $\tilde{a}_{A \alpha}(\mathbf{P}), \tilde{a}_{A \alpha}^{+}(\mathbf{P})$. The essence of this problem is to construct a dynamic mapping of superoperators $\hat{a}_{A \alpha}(\mathbf{P}), \hat{a}_{A \alpha}^{+}(\mathbf{P})$ and $\tilde{a}_{A \alpha}(\mathbf{P}), \tilde{a}_{A \alpha}^{+}(\mathbf{P})$ by some superoperators of "quasiparticles", for which the relevant thermo-vacuum state is the ground one.

In the next step, we will construct such annihilation and creation superoperators, for which the relevant thermo-vacuum state vector is a vacuum state. Analyzing the structure of relevant statistical superoperator (28), one can mark out some part that would correspond to the system of non-interacting quantum $A$-particles. Let us write $\hat{\varrho}_{\text {rel }}(t)$ in an evident form and separate terms that are connected with the interaction energy between the particles:

$$
\begin{gathered}
\hat{\varrho}_{r e l}(t)=\exp \left\{-\Phi^{*}(t)-\int \mathrm{d} \mathbf{r} \beta(\mathbf{r} ; t)\right. \\
\left.\times \sum_{A, \alpha} \int \frac{\mathrm{d} \mathbf{p}}{(2 \pi \hbar)^{3}}\left[\frac{\mathbf{p}^{2}}{2 m_{A}} \hat{n}_{A \alpha}(x)-\mu_{A \alpha}(x ; t) \hat{n}_{A \alpha}(x)\right]-\int \mathrm{d} \mathbf{r} \beta(\mathbf{r} ; t) \hat{H}_{\text {int }}(\mathbf{r})\right\} .
\end{gathered}
$$


Using operator equality ( $A$ and $B$ are some operators):

$$
\mathrm{e}^{A+B}=\left[1+\int_{0}^{1} \mathrm{~d} \tau \mathrm{e}^{\tau(A+B)} B \mathrm{e}^{-\tau A}\right] \mathrm{e}^{A},
$$

the relation for $\hat{\underline{e}}_{\text {rel }}(t)$ can be rewritten in the following form:

$$
\hat{\varrho}_{r e l}(t)=\left[1-\int \mathrm{d} \mathbf{r} \beta(\mathbf{r} ; t) \int_{0}^{1} \mathrm{~d} \tau \hat{\varrho}_{r e l}^{\tau}(t) \hat{H}_{\text {int }}(\mathbf{r})\left(\hat{\varrho}_{r e l}^{0}(t)\right)^{-\tau}\right] \hat{\varrho}_{r e l}^{0}(t),
$$

where:

$$
\hat{\varrho}_{r e l}^{0}(t)=\exp \left\{\Phi(t)-\int \mathrm{d} \mathbf{r} \beta(\mathbf{r} ; t) \sum_{A, \alpha} \int \frac{\mathrm{d} \mathbf{p}}{(2 \pi \hbar)^{3}}\left[\frac{p^{2}}{2 m_{A}} \hat{n}_{A \alpha}(x)-\mu_{A \alpha}(x ; t) \hat{n}_{A \alpha}(x)\right]\right\},
$$

or:

$$
\hat{\varrho}_{\text {rel }}^{0}(t)=\exp \left\{\Phi(t)-\int \mathrm{d} \mathbf{r} \beta(\mathbf{r} ; t) \sum_{A, \alpha} \int \frac{\mathrm{d} \mathbf{p}}{(2 \pi \hbar)^{3}} b_{A \alpha}(x ; t) \hat{n}_{A \alpha}(x)\right\},
$$

where $b_{A \alpha}(x ; t)=\left[\frac{\mathbf{p}^{2}}{2 m_{A}}-\mu_{A \alpha}(x ; t)\right]$. Relevant statistical superoperator $\hat{\varrho}_{r e l}^{0}(t)$ is bilinear on annihilation and creation superoperators $\hat{a}_{A \alpha}(\mathbf{P})$ and $\hat{a}_{A \alpha}^{+}(\mathbf{P})$, as well as on the non-perturbed part of Hamiltonian $\bar{H}_{0}$. One can write the total relevant superoperator as some non-perturbed part of $\hat{\varrho}_{r e l}^{0}(t)$ and the part that describes the interaction of quantum particles in the relevant state. Further, we introduce the following designation:

$$
\hat{\varrho}_{\text {rel }}(t)=\hat{\varrho}_{\text {rel }}^{0}(t)+\hat{\varrho}_{\text {rel }}^{\prime}(t),
$$

where:

$$
\hat{\varrho}_{r e l}^{\prime}(t)=-\int \mathrm{d} \mathbf{r} \beta(\mathbf{r} ; t) \int_{0}^{1} \mathrm{~d} \tau \hat{\varrho}_{r e l}^{\tau}(t) \hat{H}_{\text {int }}(\mathbf{r})\left(\hat{\varrho}_{r e l}^{0}(t)\right)^{-\tau} \hat{\varrho}_{r e l}^{0}(t) .
$$

Relevant thermo-vacuum states $\left.\left|\hat{\varrho}_{\text {rel }}(t)\right\rangle\right\rangle$ and $\left.\left|\hat{\varrho}_{\text {rel }}^{0}(t)\right\rangle\right\rangle$ are not vacuum states for annihilation and creation superoperators $\hat{a}_{A \alpha}(\mathbf{P}), \hat{a}_{A \alpha}^{+}(\mathbf{P}), \tilde{a}_{A \alpha}(\mathbf{P}), \tilde{a}_{A \alpha}^{+}(\mathbf{P})$. However, for $\left.\left|\hat{\varrho}_{\text {rel }}^{0}(t)\right\rangle\right\rangle$, one can construct new superoperators $\hat{\gamma}_{A \alpha}(\mathbf{P}), \hat{\gamma}_{A \alpha}^{+}(\mathbf{P}), \tilde{\gamma}_{A \alpha}(\mathbf{P}), \tilde{\gamma}_{A \alpha}^{+}(\mathbf{P})$ as a linear combination of superoperators $\hat{a}_{A \alpha}(\mathbf{P})$, $\hat{a}_{A \alpha}^{+}(\mathbf{P})$ and $\tilde{a}_{A \alpha}(\mathbf{P}), \tilde{a}_{A \alpha}^{+}(\mathbf{P})$ in order to satisfy the conditions:

$$
\begin{array}{lll}
\left.\hat{\gamma}_{A \alpha}(\mathbf{P} ; t)\left|\varrho_{\text {rel }}^{0}(t)\right\rangle\right\rangle=0, & & \left\langle\langle 1| \hat{\gamma}_{A \alpha}^{+}(\mathbf{P} ; t)=0,\right. \\
\left.\tilde{\gamma}_{A \alpha}(\mathbf{P} ; t)\left|\varrho_{\text {rel }}^{0}(t)\right\rangle\right\rangle=0, & & \left\langle\langle 1| \tilde{\gamma}_{A \alpha}^{+}(\mathbf{P} ; t)=0 .\right.
\end{array}
$$

To achieve this, let us consider an action of annihilation superoperators $\hat{a}_{A \alpha}(\mathbf{P} ; t), \tilde{a}_{A \alpha}(\mathbf{P} ; t)$ on relevant state $\left.\left|\varrho_{\text {rel }}^{0}\left(t_{0}\right)\right\rangle\right\rangle$ :

$$
\begin{aligned}
& \left.\left.\hat{a}_{A \alpha}(\mathbf{P} ; t)\left|\varrho_{r e l}^{0}\left(t_{0}\right)\right\rangle\right\rangle=f_{A \alpha}\left(\mathbf{P} ; t-t_{0}\right) \tilde{a}_{A \alpha}^{+}(\mathbf{P} ; t)\left|\varrho_{r e l}^{0}\left(t_{0}\right)\right\rangle\right\rangle, \\
& \left.\left.\tilde{a}_{A \alpha}(\mathbf{P} ; t)\left|\varrho_{r e l}^{0}\left(t_{0}\right)\right\rangle\right\rangle=\sigma f_{A \alpha}\left(\mathbf{P} ; t-t_{0}\right) \hat{a}_{A \alpha}^{+}(\mathbf{P} ; t)\left|\varrho_{r e l}^{0}\left(t_{0}\right)\right\rangle\right\rangle,
\end{aligned}
$$

where superoperators $\hat{a}_{A \alpha}(\mathbf{p} ; t), \hat{a}_{A \alpha}^{+}(\mathbf{p} ; t), \tilde{a}_{A \alpha}(\mathbf{p} ; t), \hat{a}_{A \alpha}^{+}(\mathbf{p} ; t)$ are in the Heisenberg representation:

$$
\begin{array}{ll}
\hat{a}_{A \alpha}(\mathbf{P} ; t)=\mathrm{e}^{-\frac{1}{\mathrm{i} \hbar} \bar{H}_{0} t} \hat{a}_{A \alpha}(\mathbf{P}) \mathrm{e}^{\frac{1}{i} \hbar \bar{H}_{0} t}, & \tilde{a}_{A \alpha}(\mathbf{P} ; t)=\mathrm{e}^{-\frac{1}{\mathrm{i} \hbar} \bar{H}_{0} t} \tilde{a}_{A \alpha}(\mathbf{P}) \mathrm{e}^{\mathrm{e} \frac{1}{i} \bar{H}_{0} t}, \\
\hat{a}_{A \alpha}^{+}(\mathbf{P} ; t)=\mathrm{e}^{-\frac{1}{\mathrm{i} \hbar} \bar{H}_{0} t} \hat{a}_{A \alpha}^{+}(\mathbf{P}) \mathrm{e}^{\frac{1}{\mathrm{i} \hbar} \bar{H}_{0} t}, & \tilde{a}_{A \alpha}^{+}(\mathbf{P} ; t)=\mathrm{e}^{-\frac{1}{\mathrm{i} \hbar} \bar{H}_{0} t} \tilde{a}_{A \alpha}^{+}(\mathbf{P}) \mathrm{e}^{\frac{1}{\mathrm{i} \hbar} \bar{H}_{0} t},
\end{array}
$$


and satisfy commutation relations:

$$
\begin{array}{r}
{\left[\hat{a}_{A \alpha}(\mathbf{P} ; t), \hat{a}_{B \beta}^{+}\left(\mathbf{P}^{\prime} ; t\right)\right]_{\sigma}=\delta_{A, B} \delta_{\alpha, \beta} \delta\left(\mathbf{P}-\mathbf{P}^{\prime}\right),} \\
{\left[\tilde{a}_{A \alpha}(\mathbf{P} ; t), \tilde{a}_{B \beta}^{+}\left(\mathbf{P}^{\prime} ; t\right)\right]_{\sigma}=\delta_{A, B} \delta_{\alpha, \beta} \delta\left(\mathbf{P}-\mathbf{P}^{\prime}\right),} \\
{\left[\hat{a}_{A \alpha}(\mathbf{P} ; t), \tilde{a}_{B \beta}\left(\mathbf{P}^{\prime} ; t\right)\right]_{\sigma}=\left[\hat{a}_{A \alpha}^{+}(\mathbf{P} ; t), \tilde{a}_{B \beta}^{+}\left(\mathbf{P}^{\prime} ; t\right)\right]_{\sigma}=0 .}
\end{array}
$$

It is necessary to note that superoperators $\hat{H}(\mathbf{r}), \hat{n}_{A \alpha}(x)$ are built on superoperators $\hat{a}_{A \alpha}(\mathbf{p}+$ $\left.\frac{\mathbf{q}}{2}\right), \hat{a}_{A \alpha}^{+}\left(\mathbf{p}-\frac{\mathbf{q}}{2}\right), \tilde{a}_{A \alpha}\left(\mathbf{p}+\frac{\mathbf{q}}{2}\right), \tilde{a}_{A \alpha}^{+}\left(\mathbf{p}-\frac{\mathbf{q}}{2}\right)$. Therefore, for convenience, here, a unit denotation was introduced for arguments like $\mathbf{P}=\mathbf{p} \pm \frac{\mathbf{q}}{2}$. This should be taken into account in further calculations where obvious expressions are needed.

According to the general relations of $[63,64]$, we can introduce new operators $\hat{\gamma}_{A \alpha}(\mathbf{P} ; t), \hat{\gamma}_{A \alpha}^{+}(\mathbf{P} ; t)$, $\tilde{\gamma}_{A \alpha}(\mathbf{P} ; t), \tilde{\gamma}_{A \alpha}^{+}(\mathbf{P} ; t)$ via superoperators $\hat{a}_{A \alpha}(\mathbf{P} ; t), \hat{a}_{A \alpha}^{+}(\mathbf{P} ; t), \tilde{a}_{A \alpha}(\mathbf{P} ; t), \tilde{a}_{A \alpha}^{+}(\mathbf{P} ; t)$ :

$$
\begin{aligned}
& \hat{\gamma}_{A \alpha}(\mathbf{P} ; t)=\sqrt{1+\sigma n_{A \alpha}\left(\mathbf{P} ; t, t_{0}\right)}\left[\hat{a}_{A \alpha}(\mathbf{P} ; t)-\frac{n_{A \alpha}\left(\mathbf{P} ; t, t_{0}\right)}{1+\sigma n_{A \alpha}\left(\mathbf{P} ; t, t_{0}\right)} \tilde{a}_{A \alpha}^{+}(\mathbf{P} ; t)\right], \\
& \tilde{\gamma}_{A \alpha}^{+}(\mathbf{P} ; t)=\sqrt{1+\sigma n_{A \alpha}\left(\mathbf{P} ; t, t_{0}\right)}\left[\tilde{a}_{A \alpha}^{+}(\mathbf{P} ; t)-\sigma \hat{a}_{A \alpha}(\mathbf{P} ; t)\right] .
\end{aligned}
$$

The relations (48) satisfy the conditions (46). Here:

$$
\begin{aligned}
n_{A \alpha}\left(\mathbf{p}, \mathbf{q} ; t, t_{0}\right) & =n_{A \alpha}\left(\mathbf{P} ; t, t_{0}\right)=\left\langle\left\langle 1\left|\tilde{a}_{A \alpha}^{+}(\mathbf{P} ; t) \tilde{a}_{A \alpha}(\mathbf{P} ; t)\right| \varrho_{\text {rel }}^{0}\left(t_{0}\right)\right\rangle\right\rangle \\
& =\left\langle\left\langle 1\left|\tilde{a}_{A \alpha}^{+}\left(\mathbf{p}-\frac{\mathbf{q}}{2} ; t\right) \tilde{a}_{A \alpha}\left(\mathbf{p}+\frac{\mathbf{q}}{2} ; t\right)\right| \varrho_{r e l}^{0}\left(t_{0}\right)\right\rangle\right\rangle,
\end{aligned}
$$

is a relevant distribution function of $A$-particle bound states in momentum space $\mathbf{p}$, $\mathbf{q}$, which is calculated with the help of relevant thermo-vacuum state vector $\left.\left|\varrho_{r e l}^{0}\left(t_{0}\right)\right\rangle\right\rangle$ (43). Function $f_{A \alpha}\left(\mathbf{P} ; t-t_{0}\right)$ in the formulae (47) is connected with $n_{A \alpha}\left(\mathbf{P} ; t, t_{0}\right)$ by the relation:

$$
f_{A \alpha}\left(\mathbf{P} ; t-t_{0}\right)=\frac{n_{A \alpha}\left(\mathbf{P} ; t, t_{0}\right)}{1+\sigma n_{A \alpha}\left(\mathbf{P} ; t, t_{0}\right)} .
$$

Superoperators $\hat{\gamma}_{A \alpha}(\mathbf{P} ; t), \hat{\gamma}_{A \alpha}(\mathbf{P} ; t), \tilde{\gamma}_{A \alpha}^{+}(\mathbf{P} ; t)$, and $\tilde{\gamma}_{A \alpha}^{+}(\mathbf{P} ; t)$ satisfy the "canonical" commutation relations:

$$
\begin{aligned}
& {\left[\hat{\gamma}_{A \alpha}(\mathbf{P} ; t), \hat{\gamma}_{B \beta}^{+}\left(\mathbf{P}^{\prime} ; t\right)\right]_{\sigma}=\delta_{A, B} \delta_{\alpha, \beta} \delta\left(\mathbf{P}-\mathbf{P}^{\prime}\right),} \\
& \left.\tilde{\gamma}_{A \alpha}(\mathbf{P} ; t), \tilde{\gamma}_{B \beta}^{+}\left(\mathbf{P}^{\prime} ; t\right)\right]_{\sigma}=\delta_{A, B} \delta_{\alpha, \beta} \delta\left(\mathbf{P}-\mathbf{P}^{\prime}\right), \\
& {\left[\hat{\gamma}_{A \alpha}(\mathbf{P} ; t), \tilde{\gamma}_{B \beta}\left(\mathbf{P}^{\prime} ; t\right)\right]_{\sigma}=\left[\hat{\gamma}_{A \alpha}^{+}(\mathbf{P} ; t), \tilde{\gamma}_{B \beta}^{+}\left(\mathbf{P}^{\prime} ; t\right)\right]_{\sigma}=0 .}
\end{aligned}
$$

Inversed transformations to superoperators $\hat{a}_{A \alpha}(\mathbf{P} ; t), \tilde{a}_{A \alpha}^{+}(\mathbf{P} ; t)$ are easily obtained from (48):

$$
\begin{aligned}
& \hat{a}_{A \alpha}(\mathbf{P} ; t)=\sqrt{1+\sigma n_{A \alpha}\left(\mathbf{P} ; t, t_{0}\right)}\left[\hat{\gamma}_{A \alpha}(\mathbf{P} ; t)+\frac{n_{A \alpha}\left(\mathbf{P} ; t, t_{0}\right)}{1+\sigma n_{A \alpha}\left(\mathbf{P} ; t, t_{0}\right)} \tilde{\gamma}_{A \alpha}^{+}(\mathbf{P} ; t)\right], \\
& \tilde{a}_{A \alpha}^{+}(\mathbf{P} ; t)=\sqrt{1+\sigma n_{A \alpha}\left(\mathbf{P} ; t, t_{0}\right)}\left[\tilde{\gamma}_{A \alpha}^{+}(\mathbf{P} ; t)+\sigma \hat{\gamma}_{A \alpha}(\mathbf{P} ; t)\right] .
\end{aligned}
$$

$\hat{\gamma}_{A \alpha}(\mathbf{P} ; t), \hat{\gamma}_{A \alpha}^{+}(\mathbf{P} ; t), \tilde{\gamma}_{A \alpha}(\mathbf{P} ; t), \tilde{\gamma}_{A \alpha}^{+}(\mathbf{P} ; t)$ could be defined as some operators of annihilation and creation of $A$-quasiparticle bound states, for which relevant thermo-vacuum state $\left.\left|\varrho_{\text {rel }}^{0}\left(t_{0}\right)\right\rangle\right\rangle$ (43) is a vacuum state. In such a way, we obtained relations of dynamical reflection of superoperators $\hat{a}_{A \alpha}(\mathbf{P} ; t), \hat{a}_{A \alpha}^{+}(\mathbf{P} ; t), \tilde{a}_{A \alpha}(\mathbf{P} ; t), \tilde{a}_{A \alpha}^{+}(\mathbf{P} ; t)$ to new superoperators of "quasiparticles" $\hat{\gamma}_{A \alpha}(\mathbf{P} ; t), \hat{\gamma}_{A \alpha}^{+}(\mathbf{P} ; t)$, $\tilde{\gamma}_{A \alpha}(\mathbf{P} ; t), \tilde{\gamma}_{A \alpha}^{+}(\mathbf{P} ; t)$. Now, we can consider some particular strategy for the calculation of the transport cores by switching to the superoperators of "quasiparticles" $\hat{\gamma}_{A \alpha}(\mathbf{P} ; t), \hat{\gamma}_{A \alpha}^{+}(\mathbf{P} ; t), \tilde{\gamma}_{A \alpha}(\mathbf{P} ; t), \tilde{\gamma}_{A \alpha}^{+}(\mathbf{P} ; t)$. 
Here, we can use series expansions over the interaction of the relevant superoperator (44). These issues require a separate, detailed study.

A set of transport Equations (32) and (33) together with dynamical reflections (48) and (50) of superoperators in the thermo-field space constitute the basis for a consistent description of the kinetics and hydrodynamics of a dense quantum system with strongly-bound states. Both strong and weak nonequilibrium processes of a nuclear matter can be investigated using this approach, in which the particle interaction is characterized by strongly-bound states, taking into account their nuclear nature [3-6].

Weak nonequilibrium processes can be described when the fluctuations of the parameters $\delta \beta(\mathbf{r} ; t)=\beta(\mathbf{r} ; t)-\beta, \delta \mu_{A \alpha}(x ; t)=\mu_{A \alpha}(x ; t)-\mu_{A \alpha}$ are small, where $\beta$ and $\mu_{A \alpha}$ are equilibrium values for temperature and chemical potential, respectively. In this case, the system of Equations (32) and (33) will have a similar structure, but is closed with respect to $\left\langle\left\langle 1\left|\delta \hat{n}_{A \alpha}(x)\right| \varrho(t)\right\rangle\right\rangle,\langle\langle 1|\delta \hat{H}(\mathbf{r})| \varrho(t)\rangle\rangle$, where $\left.\delta \hat{n}_{A \alpha}(x)=\hat{n}_{A \alpha}(x)-\left\langle\left\langle 1\left|\hat{n}_{A \alpha}(x)\right| \varrho_{0}\right\rangle\right\rangle, \delta \hat{H}(\mathbf{r})=\hat{H}(\mathbf{r})-\left\langle\left\langle 1|\hat{H}(\mathbf{r})| \varrho_{0}\right\rangle\right\rangle,\left|\varrho_{0}\right\rangle\right\rangle$ is the equilibrium thermo vacuum state vector of the systems.

In addition, by designing a system of equations on moments $1, \mathbf{P}$ of the distribution function, we obtain, respectively, the equation of the thermo-field hydrodynamic for the dense quantum-field systems. These questions require separate consideration and will be investigated in future work.

\section{Conclusions}

We generalized the nonequilibrium thermo-field dynamics in the framework of Zubarev's nonequilibrium statistical operator method [63] within the framework of Renyi statistics. The non-Markov transport equations in the thermo-field presentation in Renyi statistics are obtained, which can be used to describe the nonequilibrium processes in quantum Bose and Fermi systems. In the case of $q \rightarrow 1$ [72,73], when Renyi statistics are transformed into non-extensive Tsallis statistics, we obtain the corresponding generalized transport equations with non-additive entropy for the system. Based on this approach and Gibbs statistics, the generalized equations of the consistent description of kinetics and hydrodynamics for dense quantum field systems with strongly-bound states were obtained. We obtained the relations of the dynamic mapping of the superoperators $\hat{a}_{A \alpha}(\mathbf{P} ; t), \hat{a}_{A \alpha}^{+}(\mathbf{P} ; t)$, $\tilde{a}_{A \alpha}(\mathbf{P} ; t), \tilde{a}_{A \alpha}^{+}(\mathbf{P} ; t)$ with the new superoperators of "quasiparticles" $\hat{\gamma}_{A \alpha}(\mathbf{P} ; t), \hat{\gamma}_{A \alpha}^{+}(\mathbf{P} ; t), \tilde{\gamma}_{A \alpha}(\mathbf{P} ; t)$, $\tilde{\gamma}_{A \alpha}^{+}(\mathbf{P} ; t)$, for which the relevant thermo vacuum state of the non-interacting particles is the ground one. This is important for the calculation of the transport cores.

Using this approach, one can investigate both strong and weak nonequilibrium processes of nuclear matter, when the interaction between particles of the latter is characterized by strongly-bound states of an internucleon nature [4,5].

Author Contributions: Conceptualization, M.T.; Methodology, M.T.; Investigation, M.T., P.H.; Writing-Original Draft Preparation, M.T., P.H.; Writing—Review \& Editing, M.T., P.H.

Funding: This research received no external funding.

Conflicts of Interest: The authors declare no conflict of interest.

\section{References}

1. Röpke, G. Nuclear matter EoS including few-nucleon correlations. Nuovo Cimento C 2016, 39, 392.

2. Röpke, G. Correlations and clustering in dilute matter. In Nuclear Particle Correlations and Cluster Physics; Schröder, W., Ed.; World Scientific: Singapore, 2017; Chapter 2, pp. 31-69.

3. Negele, J.W. The mean field theory of nuclear structure and dynamics. Rev. Mod. Phys. 1982, 54, $913-1015$. [CrossRef]

4. Toneev, V.D.; Shultz, H.; Gudima,K.K.; Röpke, G. Towards study of hot and dense nuclear matter in heavy ion collisions. Phys. Elem. Part. Nucl. (Part. Nucl.) 1986, 17, 1093-1172. (In Russian)

5. Röpke, G.; Shultz, H.; Gudima, K.K.; Toneev, V.D. Dynamical approaches to heavy-ion collision in intermediate energy region. Phys. Elem. Part. Nucl. (Part. Nucl.) 1990, 21, 364-418. (In Russian) 
6. McLerran, L. The physics of the quark-gluon plasma. Rev. Mod. Phys. 1986, 58, 1021-1064. [CrossRef]

7. Blättel, B.; Koch, V.; Mosel, U. Transport-theoretical analysis of relativistic heavy-ion collisions. Rep. Prog. Phys. 1993, 56. [CrossRef]

8. Mrówczyńsky, S. Quark-Qluon Plasma; World Scientific: Singapore, 1990.

9. Cleymans, J.; Gavai, R.V.; Suhonen, E. Quarks and gluons at high temperatures and densities. Phys. Rep. 1986, 130, 217-292. [CrossRef]

10. Jacob, M.; Satz, H. Quark Matter Formation and Heavy Ion Collisions; World Scientific: Singapore, 1982.

11. McLerran, L. Probes of the quark-gluon plasma as it might be produced in ultra-relativistic nuclear collisions. Acta Phys. Pol. B 1985, 16, 669-682.

12. Shuryak, E.V. What RHIC experiments and theory tell us about properties of quark-gluon plasma? Nucl. Phys. A 2005, 750, 64-83. [CrossRef]

13. Schuryak, E. Strongly coupled quark-gluon plasma in heavy ion collisions. Rev. Mod. Phys. 2017, 89, 035001. [CrossRef]

14. Jaiswal, A.; Roy, V. Relativistic hydrodynamics in heavy-ion collisions: General aspects and recent developments. Adv. High Energy Phys. 2016, 2016, 9623034. [CrossRef]

15. Florkowski, W.; Heller, M.P.; Spalinski, M. New theories of relativistic hydrodynamics in the LHC era. Rep. Prog. Phys. 2018, 81, 046001. [CrossRef] [PubMed]

16. Li, Y. A flow paradigm in heavy-ion collisions. arXiv 2018, arXiv:1712.04580.

17. Hirino, T.; van der Kolk, N.; Bilandzic, A. Hydrodynamics and Flow. Lect. Notes Phys. 2010, 785, 139-178._4. [CrossRef]

18. Florkowski, W. Hydrodynamic description of ultrarelativistic heavy-ion collisions. arXiv 2017, arXiv:1712.05162.

19. Röpke, G.; Shlomo, S.; Bonasera, A.; Natowitz, J.B.; Yennello, S.J.; McIntosh, A.B.; Mabiala, J.; Qin, L.; Kowalski, S.; Hagel, K.; et al. Density determinations in heavy ion collisions. Phys. Rev. C 2013, 88, 024609. [CrossRef]

20. Hempel, M.; Hagel, K.; Natowitz, J.; Röpke, G.; Typel, S. Constraining supernova equations of state with equilibrium constants from heavy-ion collisions. Phys. Rev. C 2015, 91, 045805. [CrossRef]

21. Bastian, N.-U.F.; Blaschke, D.; Fischer, T.; Röpke, G. Nowards a Unified Quark-Hadron-Matter Equation of State for Applications in Astrophysics and Heavy-Ion Collisions. Universe 2018, 4, 67. [CrossRef]

22. Elze, H.-T.; Heinz, U. Quark-gluon transport theory. Phys. Rep. 1989, 183, 81-135. [CrossRef]

23. Lee, T.D. The strongly interacting quark-gluon plasma and future physics. Nucl. Phys. A 2005, 750, 1-8. [CrossRef]

24. Gyulassy, M.; McLerran, I. New forms of QCD matter discovered at RHIC. Nucl. Phys. A 2005, 750, 30-63. [CrossRef]

25. Romatschke, P. Do nuclear collisions create a locally equilibrated quark-gluon plasma? Eur. Phys. J. 2017, 77, 21. [CrossRef]

26. Romatschke, P. Relativistic Fluid Dynamics Far From Local Equilibrium. Phys. Rev. Lett. 2018, 120, 012301. [CrossRef] [PubMed]

27. Baier, R.; Romatschke, P.; Son, D.T.; Starinets, A.O.; Stephanov, M.A. Relativistic viscous hydrodynamics, conformal invariance, and holography. J. High Energy Phys. 2008, 4, 100. [CrossRef]

28. Zubarev, D.N.; Morozov, V.G. Formulation of boundary conditions for the BBGRY hierarchy with allowance for local conservation laws. Theor. Math. Phys. 1984, 60, 814-820. [CrossRef]

29. Zubarev, D.N.; Morozov, V.G.; Omelyan, I.P.; Tokarchuk, M.V. Kinetic equations for dense gases and liquids. Theor. Math. Phys. 1991, 87, 412-424. [CrossRef]

30. Zubarev, D.N.; Morozov, V.G.; Omelyan, I.P.; Tokarchuk, M.V. Unification of the kinetic and hydrodynamics approaches in theory of dense gases and liquids. Theor. Math. Phys. 1993, 96, 997-1012. [CrossRef]

31. Markiv, B.; Omelyan, I.; Tokarchuk, M. Consistent Description of Kinetics and Hydrodynamics of Weakly Nonequilibrium Processes in Simple Liquids. J. Stat. Phys. 2014, 155, 843-866. [CrossRef]

32. Alberico, W.M.; Lavagno, A.; Quarati, P. Nonextensive statistics, fluctuations and correlations in high energy nuclear collisions. Eur. Phys. J. C 2000, 12, 499-506. [CrossRef]

33. Biro, T.S.; Molnar, E. Fluids dynamical equations and transport coefficients of relativistic gases with non-extensive statistics. Phys. Rev. C 2012, 85, 024905. [CrossRef]

34. Shen, K.-M.; Biro, T.S.; Wang, E.-K. Different non-extensive models for heavy-ion collisions. Physica A 2018, 492, 2353-2360. [CrossRef] 
35. Osada, T.; Wilk, G. Nonextensive/Dissipative Correspondence in Relativistic Hydrodynamics. Prog. Theor. Phys. Suppl. 2008, 174, 168-172. [CrossRef]

36. Osada, T.; Wilk, G. Erratum: Nonextensive hydrodynamics for relativistic heavy-ion collisions. Phys. Rev. C 2008, 77, 044903. [CrossRef]

37. Osada, T.; Wilk, G. Nonextensive perfect hydrodynamics-A model of dissipative relativistic hydrodynamics? Cent. Eur. J. Phys. 2009, 7, 432-443. [CrossRef]

38. Osada, T. Relativistic hydrodynamical model in the presence of long-range correlations. Phys. Rev. C 2010, 81, 024907. [CrossRef]

39. Lavagno, A. Relativistic nonextensive thermodynamics. Phys. Lett. A 2002, 301, 13-18. [CrossRef]

40. Gianpiero,G.; Lavagno, A.; Pigato, D. Nonextensive statistical effects in the quark-gluon plasma formation at relativistic heavy-ion collisions energies. Cent. Eur. J. Phys. 2012, 10, 594-601. [CrossRef]

41. Lavagno, A.; Pigato, D. Nonextensive statistical effects and strangeness production in hot and dense nuclear matter. J. Phys. G Nucl. Part. 2012, 39, 125106. [CrossRef]

42. Lavagno, A.; Pigato, D. Nonextensive nuclear liquid-gas phase transition. Physica A 2013, 392, 5164-5171. [CrossRef]

43. Wilk, G.; Wlodarczyk, Z. Temperature oscillations and sound waves in hadronic matter. Physica A 2017, 486, 579-586. [CrossRef]

44. Zubarev, D.N.; Prozorkevich, A.V.; Smolyanskii, S.A. Derivation of nonlinear generalized equations of quantum relativistic hydrodynamics. Theor. Math. Phys. 1979, 40, 821-831. [CrossRef]

45. Prozorkevich, A.V.; Samorodov, V.L.; Smolyanskii, S.A. Quantum relativistic hydrodynamics of systems with broken symmetry. I. Local-equilibrium state. Theor. Math. Phys. 1982, 52, 920-926. [CrossRef]

46. Smolyanskii, S.A. Quantum relativistic hydrodynamics of systems with broken symmetry II. Nonequilibrium state. Theor. Math. Phys. 1982, 52, 809-814. [CrossRef]

47. Hosoya, A.; Sakagami, M.A.; Takao, M. Nonequilibrium thermodynamics in field theory: Transport coefficients. Ann. Phys. 1984, 154, 229-252. [CrossRef]

48. Becattini, F.; Tinti, L. Nonequilibrium thermodynamical inequivalence of quantum stress-energy and spin tensors. Phys. Rev. D 2013, 87, 025029. [CrossRef]

49. Tinti, L. Progress in Mathematical Relativity, Gravitation and Cosmology; Springer: Berlin/Heidelberg, Germany, 2014.

50. Becattini, F.; Bucciantini, L.; Grossi, E.; Tinti, L. Local thermodynamical equilibrium and the $\beta$ frame for a quantum relativistic fluid. Eur. Phys. J. C 2015, 75, 191. [CrossRef]

51. Hayata, T.; Hidaka, Y.; Nomi, T.; Hongo, M. Relativistic hydrodynamics from quantum field theory on the basis of the generalized Gibbs ensemble method. Phys. Rev. D 2015, 92, 065008. [CrossRef]

52. Harutyunyan, A.; Sedrakian, A.; Rischke, D.H. Relativistic Dissipative Fluid Dynamics from the Non-Equilibrium Statistical Operator. Particles 2018, 1, 155-165. [CrossRef]

53. Harutyunyan, A.; Sedrakian, A. Bulk Viscosity of the Hot Quark Plasma from from the Non-Equilibrium Statistical Operator. Particles 2018, 1, 212-229. [CrossRef]

54. Koide, T.; Kodama, T. Transport coefficient of non-Newtonian fluid and causal dissipative hydrodynamis. Phys. Rev. E 2008, 78, 051107. [CrossRef]

55. Huang, X.-G.; Koide, T. Shear viscosity, bulk viscosity, and relaxation times of causal dissipative relativistic fluid-dynamics at finite temperature and chemical potential. Nucl. Phys. A 2013, 889, 73-92. [CrossRef]

56. Morozov, V.G.; Ropke, G.; Holl, A. Kinetic Theory of Quantum Electrodynamic Plasma in a Strong Electromagnetic Field: I. The Covariant Formalism. Theor. Math. Phys. 2002, 131, 812-831. [CrossRef]

57. Morozov, V.G.; Ropke, G.; Holl, A. Kinetic Theory of Quantum Electrodynamic Plasma in a Strong Electromagnetic Field: II. The Covariant Mean-Field Approximation. Theor. Math. Phys. 2002, 132, 1029-1042. [CrossRef]

58. Blaizot, J.-P.; Li, Y. Onset of hydrodynamics for a quark-gluon plasma from the evolution of momentsof distribution functions. J. High Energy Phys. 2017, 161. [CrossRef]

59. Tinti, L.; Jaiswal, A.; Ryblewski, R. Quasiparticle second-order viscous hydrodynamics from kinetic theory. Phys. Rev. D 2017, 95, 054007. [CrossRef]

60. Arimitsu, T.; Umezawa, H. A General Formulation of Nonequilibrium Thermo Field Dynamics. Progr. Theor. Phys. 1985, 74, 429-432. [CrossRef] 
61. Arimitsu, T.; Umezawa, H. Non-Equilibrium Thermo Field Dynamics. Progr. Theor. Phys. 1987, 77, 32-52. [CrossRef]

62. Arimitsu, T. A canonical formalism of dissipative quantum systems. Non-equilibrium thermofield dynamics. Condens. Matter Phys. 1994, 4, 26-88. [CrossRef]

63. Zubarev, D.N.; Tokarchuk, M.V. Nonequilibrium thermofield dynamics and the nonequilibrium statistical operator method I. Basic relations. Theor. Math. Phys. 1991, 88, 876-893. [CrossRef]

64. Tokarchuk, M.V.; Arimitsu, T.; Kobryn, A.E. Thermo field hydrodynamic and kinetic equations of dense quantum nuclear systems. Condens. Matter Phys. 1998, 1, 605-642. [CrossRef]

65. Glushak, P.A.; Markiv, B.B.; Tokarchuk, M.V. Zubarev's Nonequilibrium Statistical Operator Method in the Generalized Statistics of Multiparticle Systems. Theor. Math. Phys. 2018, 194, 57-73. [CrossRef]

66. Takahashi, Y.; Umezawa, H. Thermo field dynamics. Collect. Phenom. 1975, 2, 55-80; reprinted in Int. J. Mod. Phys. B 1996, 10, 1755-1805. [CrossRef]

67. Umezawa, H.; Matsumoto, H.; Tachiki, M. Thermo Field Dynamics and Condensed States; North-Holland: Amsterdam, The Netherlands, 1982.

68. Zubarev, D.N. Nonequilibrium Statistical Thermodynamics; Consultant Bureau: New York, NY, USA, 1974.

69. Zubarev, D.N.; Morozov, V.G.; Ropke, G. Statistical Mechanics of Nonequilibrium Processes; Akademie Verlag: Berlin, Germany, 1996; Volume 1.

70. Zubarev, D.N.; Morozov, V.G.; Ropke, G. Statistical Mechanics of Nonequilibrium Processes; Akademie Verlag: Berlin, Germany, 1997; Volume 2.

71. Markiv, B.B.; Tokarchuk, R.M.; Kostrobij, P.P.; Tokarchuk, M.V. Nonequilibrium statistical operator method in Renyi statistics. Physica A 2011, 390, 785-791. [CrossRef]

72. Rudoi, Y.G. Generalized Informational Entropy and Noncanonical Distribution in Equilibrium Statistical Mechanics. Theor. Math. Phys. 2003, 135, 451-496.

73. Bashkirov, A.G. On maximum entropy principle, superstatistics, power-law distribution and Renyi parameter. Physica A 2004, 340, 153-162. [CrossRef]

(C) 2018 by the authors. Licensee MDPI, Basel, Switzerland. This article is an open access article distributed under the terms and conditions of the Creative Commons Attribution (CC BY) license (http://creativecommons.org/licenses/by/4.0/). 